\title{
Nanostructured Thin Films for Fluorescence Enhancement
}

\author{
S. Lévêque-Fort ${ }^{a}$, E. Fort ${ }^{b}$, E. Le Moal ${ }^{a, b}$, J.-P. Lacharme ${ }^{b}$, M.-P. Fontaine-Aupart ${ }^{a}$ \\ and C. Ricolleau ${ }^{b}$ \\ ${ }^{a}$ Laboratoire de Photophysique Moléculaire, CNRS UPR 3361, Orsay, France \\ ${ }^{b}$ Laboratoire de Physique du Solide \\ and "Matériaux et Phénomènes Quantiques", Fédération de Recherche CNRS 2437 \\ ESPCI, 10 rue Vauquelin, \\ 75005 Paris Cedex, France
}

\begin{abstract}
Fluorescence is widely used as a spectroscopic tool or for biomedical imaging, in particular for DNA chips. Nanostructured metallic substrates permit to locally enhance the fluorescence signal which offer the possibility both to detect very small fluorophore concentrations and to trace precisely the biomarkers. We have developed substrates made of silver or gold nanoparticles covered with a spacer layer of alumina. Double metallic and dielectric gradients permit to directly map the fluorescence enhancement factor and to determine the best condition for maximum enhancement. One and two photons excitations are studied. Fluorescence enhancement reaches two orders of magnitudes. Lifetime measurements reveal additional information on the decay channels induced by the nanoparticle presence.
\end{abstract}

Keywords: Fluorescence enhancement, metallic nanoparticles, surface plasmon

\section{INTRODUCTION}

Fluorescence is a powerful and widely used technique in biological studies. It has been successfully used as a diagnostic tool for tissue analysis or to provide an easy and quick read out method. One of the main limitations in most of the recent studies and applications comes from the need to detect very low molecular concentrations, with the extreme case of single molecule detection. Another, current trend $n$ biological issues is the need for a precise localization of the bio-markers, in particular for diffusion studies for which it is necessary to accurately trace the active molecules.

In DNA chips applications, both aspects are important :

1. precise localization is needed to increase the signal to noise ratio(for instance to differentiate the hybridized DNA strand from the unwashed strands) or for high-density DNA chips;

2. detection of very low concentrations: for instance in medical applications for which the quantity is limited by the biopsy.

Nanostructured samples offer a good and un-expensive way to solve this technological bottleneck. It is well known that metallic nanoparticles, made of silver and gold for instance, can enhance the fluorescence signal of conventional fluorophores by many orders of magnitude. When excited at their plasmon wavelength, the metallic nanoparticles act as a local antenna. Therefore it is highly interesting to develop nanostructured substrates which increase the signal and allow to keep commonly used detection set-up.

In the next section, we present the basic ideas governing the nanoparticle-fluorophore interaction. fection 3 is devoted to the description of the nanostructured sample preparation and of the experimental setups, results and discussions are gathered in the last section.

Further author information: (Send correspondence to S.L.F.)

s.L.F.: E-mail: sandrine.fort@ppm.u-psud.fr. Telephone: +33 (0)1691582 34 


\section{SIMPLE MODEL OF NANOPARTICLE-FLUOROPHORE INTERACTIONS}

The enhancement of the fluorescence intensity of the fluorophores in the vicinity of metallic nanoparficules can be expressed by the enhancement factor $Y$ which is defined as the ratio of the observed fluorescence intensity of the dye molecules on the nanostructured substrate relative to the intensity of an identical dye layer on an inert quartz substrate at the same laser illumination conditions. Using the model of Gersten and Nitzan $Y$ is the product of two factors ${ }^{1}$ :

$$
Y=Y_{\text {exc. }}\left(\omega_{\text {exc }}\right) Y_{\text {emis. }}\left(\omega_{f l u}\right)
$$

The first factor $Y_{\text {exc. }}\left(\omega_{\text {exc }}\right)$ describes the excitation intensity sensed by the dye molecules enhanced py the local field around the nanoparticle. The second factor $Y_{\text {emis. }}\left(\omega_{f l u}\right)$ accounts for the partitioning of the excitation energy into the various radiative and non-radiative decay channels modified by the hanoparticles presence.

The electromagnetic response of metallic nanoparticles exibit some resonances associated with the collective oscillation of the conduction electrons in the metal (plasmon modes). These resonances induce an exaltation of the electromagnetic field in the vicinity of the nanoparticles. The local field enhancement s defined by the factor $L(\omega)$. Assuming a spheroidal shape for the nanoparticles the enhancement factor $L(\omega)$ can be calculated analytically using Mie theory ${ }^{1}$ alternatively numerical models give the exaltation field map for an arbitrary particle shape. ${ }^{2}$

The local electromagnetic field is greatly enhanced if the excitation frequency $\omega_{\text {exc }}$ corresponds to the plasmon frequency $\omega_{p l}$ of the nanoparticle. For example, $L\left(\omega_{p l}\right)$ reaches the value 140 in the equatorial plane of a silver prolate spheroid (with polar half axis $4 \mathrm{~nm}$ and equatorial half axis $10 \mathrm{~nm}$ ). ${ }^{3}$ The field s enhanced on a characteristic length of about the size of the particle. Besides, the exaltation is more mportant near the tip shaped surfaces (antenna effect).

The excitation factor $Y_{\text {exc. }}\left(\omega_{\text {exc }}\right)$ in the equation 1 is equal to $\left|L\left(\omega_{\text {exc }}\right)\right|^{2}$ for the one photon excitation fluorescence and to $\left|L\left(\omega_{e x c}\right)\right|^{4}$ for the two photon excitation. Since the field enhancement can reach high values, the two photon fluorescence can thus give much larger enhancement than the one photon fluorescence. Again, to obtain large enhancement factors, the plasmon frequency needs to be adjusted to the excitation frequency of the fluorophore.

The enhancement of the radiative yield described by the second factor of equation 1 depends on the additional radiative and non-radiative decay processes induced by the nanoparticle presence. It can be defined for a dye molecule at a distance $r$ of the nanoparticle as $Y_{\text {emis. }}=\gamma(r) / \gamma(\infty)$ where $\gamma(r)$ is the fluorescence quantum yield of the dye molecule at a distance $r$ from the particle and $\gamma(\infty)$ corresponds to the fluorophore in free space. The fluorescence quantum yield is defined as follows:

$$
\gamma(r)=\frac{k_{r}+\Gamma_{r}(r)}{k_{r}+\Gamma_{r}(r)+k_{n r}+\Gamma_{n r}(r)}
$$

where $k_{r}$ and $k_{n r}$ are the radiative and non-radiative decay rates of the dye molecule on an inert substrate and $\Gamma_{r}$ and $\Gamma_{n r}$ are the additional rates induced at a distance $r$ by the nanoparticle presence.

The molecular emission induces an electronic polarization in the nanoparticles which is large at the frequency of the plasmon resonance. The in-phase dipolar component of the response increases the total emission dipole resulting in an increase in the radiative emission rate. The electromagnetic interactions feflect the coupling of the molecular dipole with the island dipole to the emitted field. The enhancement of the radiative emission rate near the metallic island satisfy $k_{r}+\Gamma_{r}(r)=\left|L\left(\omega_{f l}\right)\right|^{2} k_{r}{ }^{1}$

The additional non-radiative channels associated with the nanoparticle presence arise from the outof-phase dipolar component of the island and from the non-radiating higher poles induced in the island py the molecular dipole. The additional nonradiative decay rates associated with the particule presence vary like $\Gamma_{n r}(r) \propto r^{-3}$ and reach very large values at very small distances of the nanoparticles. ${ }^{4}$ These honradiating processes are directly responsible for the quenching of the fluorescence for very small 
hanoparticle-molecule distances where we would expect the largest enhancements from the emission yield contribution alone.

The fluorescence emission yield is very different for molecules with high and low fluorescence yield. For high fluorescence yield, i.e. for $\gamma(\infty) \approx 1$, the emission yield cannot contribute to the enhancement since $\gamma(r)$ maximum value is 1 . Since this value can be much smaller due to additional nonradiative decay channels, the fluorescence can even be damped by emission yield. For low fluorescence yield however, i.e. for $\gamma(\infty) \ll 1$, the emission yield can be as large as $Y_{\text {emis }} \simeq 1 / \gamma(\infty) .{ }^{5}$ This is the case when there exist an intermediate nanoparticle-fluorophore distance $r_{0}$ where additional radiative decay channels are numerous compared to nonradiative ones, i.e. $\gamma\left(r_{0}\right) \approx 1$. It means that the total fluorescence enhancement induced by the vicinity of the nanoparticle is even more important for molecules which ptherwise do not fluoresce much.

\section{SAMPLE PREPARATION AND EXPERIMENTAL SETUPS}

The samples are prepared under ultra high vacuum conditions on conventional coverslips cleaned by standard chemical procedures and oxygen plasma cleaning.

The alumina deposition is performed by pulsed laser deposition (PLD) technique performed by a KrF excimer laser using an alumina target. The average deposition rate is $0.4 \AA / \mathrm{s}$ for a pulse repetition rate of $4 \mathrm{~Hz}$. The alumina film obtained by this technique is continuous, amorphous and dense to prevent dye diffusion.

Silver is deposited by thermal evaporation technique at a rate of $10 \AA / \mathrm{mn}$. The metallic nanostrucfured layer is deposited onto an underlayer of alumina of about $2 \mathrm{~nm}$ to insure high sample reproducibility concerning the nanoparticle shape and distribution. An additional alumina spacerlayer is deposited on fop of the nanoparticles to tune the nanoparticle-fluorophore distance.

Both alumina and silver depositions are controlled by a quartz balance thickness monitor. In this paper, the nominal thickness given to define the amount of silver deposited corresponds to the thickness of the equivalent continuous film. Masks allow to hide successively parts of the sample during the deposition process and to vary the deposited thickness to produce samples with gradients. It is thus possible to obtain directly on the same sample the influence of silver and spacerlayer thickness on the fluorescence enhancement factor.

The shape and size distributions of the nanoparticles depend on the deposition parameters (thickness, temperature, flux,...). The nanostructured sample are characterized by transmission electron microscopy (TEM) images, obtained using a JEOL 2000 EX electron microscope operating at $200 \mathrm{kV}$. The image fesolution is about $0.5 \mathrm{~nm}$. Since the plasmon resonance depends drastically on the shape of the particles, the optical absorption spectra (acquired with a Cary 500 differential optical spectrometer) give precious nformation on the nanostructured substrate.

The dye molecules are deposited onto the nanostructured thin films by spin coating or by thermal evaporation under vacuum to obtain homogeneous deposition.

The two photon fluorescence set-up is based on a inverted microscope (Zeiss axiovert 135). The laser peam from the Ti:Sa laser (100 fs, $76 \mathrm{MHz}$, attenuated to less than $10 \mathrm{~mW}$ ) is focussed on the sample through an immersion oil objective (Zeiss x63 NA 1.4). The fluorescence emission is collected by the same objective and transmitted through a dichroic mirror along the emission path. Fluorescence is then detected by a microchannel plate associated to a conventional Time Correlated Single Photon Counting system (TCSPC). The temporal resolution of our system is 30 ps. $^{6}$

The fluorescent intensity images of the samples are obtained using a standard commercial scanner. 


\section{RESULTS AND DISCUSSION}

The position of the plasmon resonance plays a crucial role to obtain high exaltation yields. In order to achieve high enhancements it should either be adjusted to the frequency of the excitation field or alternatively to the emission frequency. The plasmon resonance wavelength are presented for silver hanostructured thin films uncovered and covered with $14 \mathrm{~nm}$ of alumina in figure 1 for various silver hominal thicknesses. The peak associated with the plasmon resonance, clearly visible in the absorption spectra, shifts from $475 \mathrm{~nm}$ to $575 \mathrm{~nm}$ for increasing silver deposition. This shift is associated with a global change in the particles aspect ratio. The aspect ratio defined as the thickness over the diameter of the nanoparticle increases with decreasing silver deposition as confirmed by TEM images shown in figure 2 .

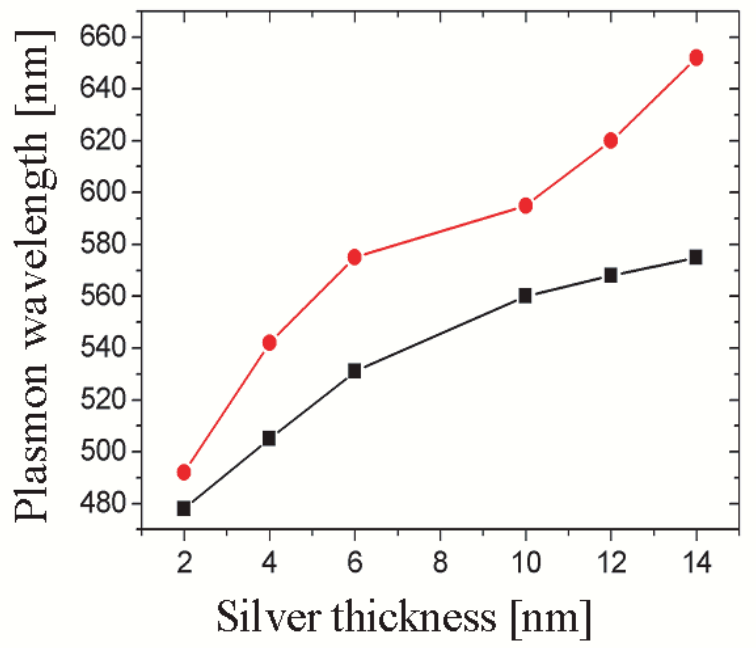

Figure 1. Resonance plasmon wavelength versus various silver nominal thicknesses uncovered (squares) and covered with $14 \mathrm{~nm}$ of alumina (circles).

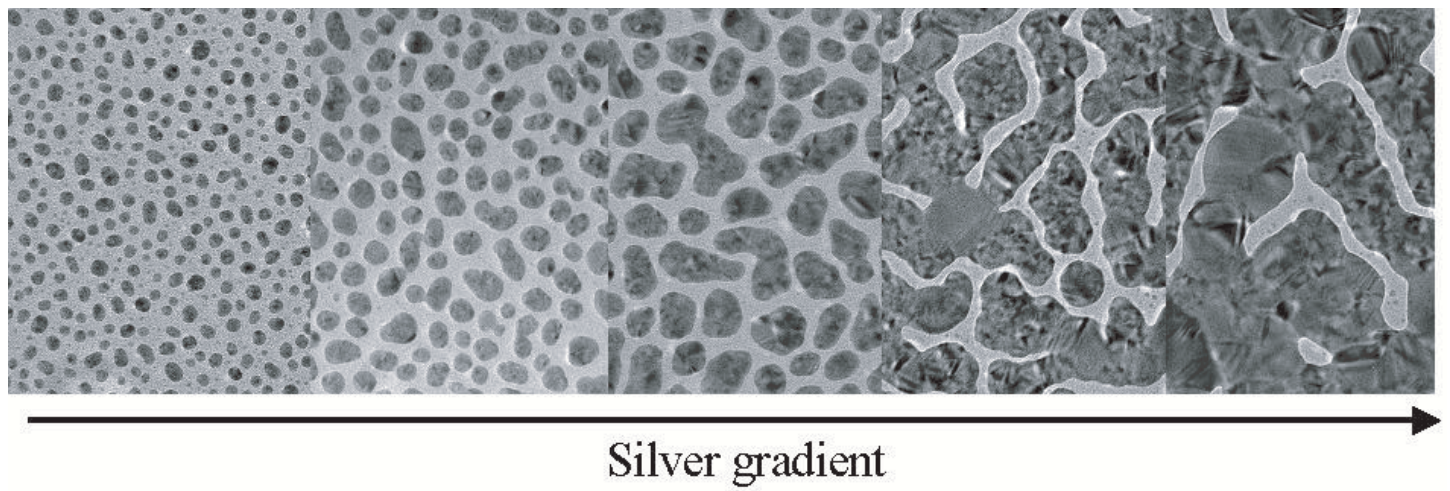

Figure 2. TEM images of the nanostructured thin films for increasing thicknesses; The height of the image is $22 \mathrm{~nm}$

The cover layer of alumina induces a redshift as presented in figure 1. The resonance plasmon $\mathrm{s}$ shifted between 490 to $650 \mathrm{~nm}$. This well known behavior originates in the changing the of the 
purrounding dielectric constant of the nanoparticle. ${ }^{7}$

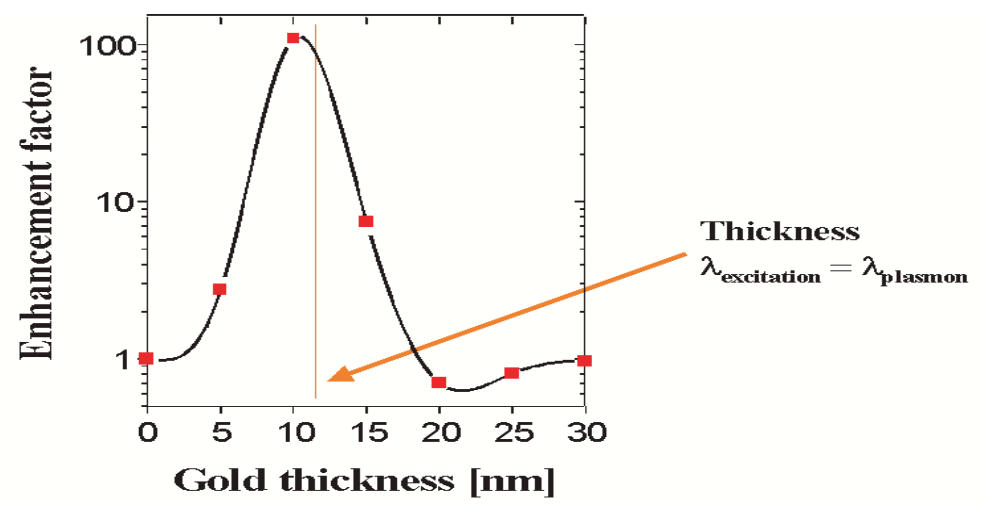

Figure 3. Fluorescence enhancement for two photon excitations obtained on the same sample with a gold gradient for 0.01 monolayer of eosine excited at $833 \mathrm{~nm}$

Figure 3 shows the influence of the plasmon position on the fluorescence enhancement for two photon fluorescence excitation at $833 \mathrm{~nm}$ for Eosine coating on a gold nanostructured thin film. The enhancement feaches the maximum value of about 100 when the plasmon resonance wavelength coincide with the excitation wavelength. As mentioned previously, enhancement is expected to occur when the plasmon wavelength is tuned at the excitation frequency to induce large local electric fields. Note that gold is more appropriate for near IR excitation wavelength as compared to silver since its plasmon resonance is $n$ this spectral range.

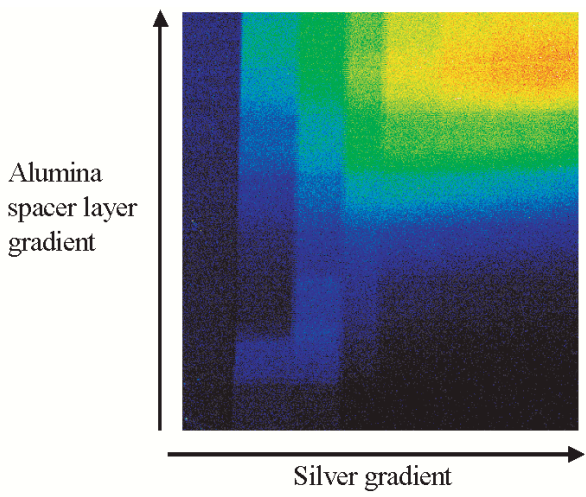

Figure 4. Fluorescence intensity image of a sample with a double alumina and silver gradient covered with 0.05 monolayer of rhodamine B. Excitation wavelength $550 \mathrm{~nm}$

Figure 4 shows the fluorescence intensity image of a sample with a double silver and alumina gradient obtain using a commercial scanner. The sample is covered with approximately 0.05 monolayer of rhodamine B excited at $550 \mathrm{~nm}$. An optimum spacer layer distance is clearly visible on the image. These samples permit an easy mapping of the fluorescence enhancement versus metallic deposition and spacer layer thickness and consequently are a quick way to determine the optimal conditions to reach a maximum signal amplification.

Figure 5 represent the enhancement factor map obtained from a single sample with a double gradient deduced from the scanner image. The sample is covered with about 0.05 monolayer of cyanine 3 . Again an optimum silver deposition and an associated alumina spacer laver are clearly visible. The fluorescence 


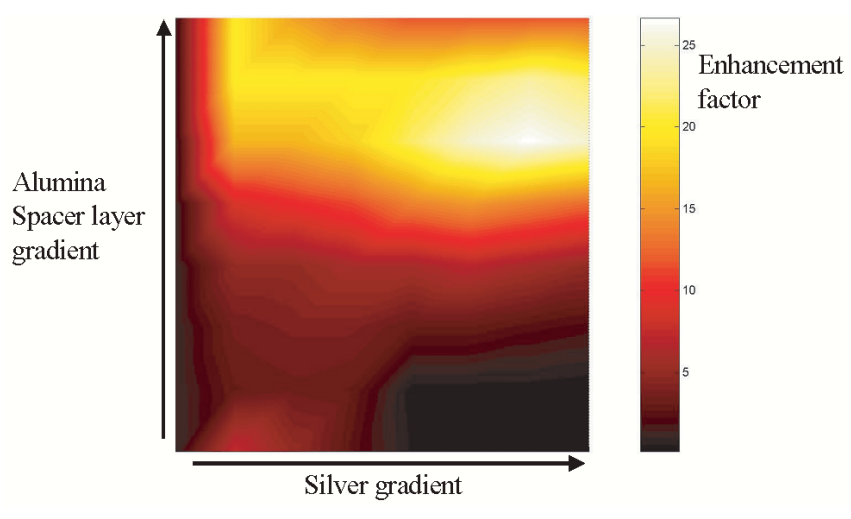

Figure 5. Fluorescence intensity enhancement obtained on a sample with a double alumina and silver gradient covered with 0.05 monolayer of cyanine 3. Excitation wavelength $550 \mathrm{~nm}$

enhancement reaches the maximum value of 25. It is noteworthy that the alumina spacer layer does not pnly act as a spacer layer but also induces a red-shift of the plasmon resonance wavelength.

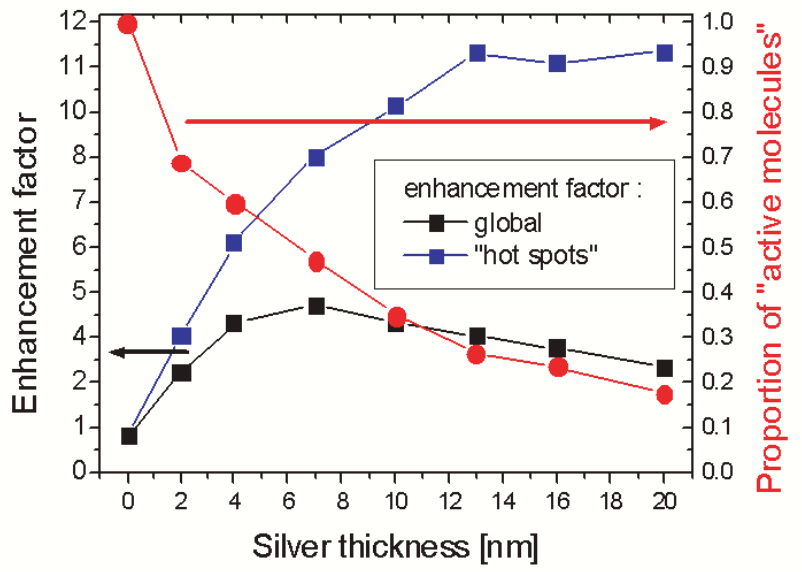

Figure 6. Global and local ("hot spot") fluorescence enhancement factor versus silver deposition. The proportion of active molecules is deduce from silver coverage measured on TEM images

The observed fluorescence enhancement on figure 5 is associated with the global enhancement of the dye layer. However, it is well known that the the local electromagnetic field is strongly enhanced in ocalized area, called "hot spot". 8 In particular, fluorescence is quenched when the dye molecules are deposited directly on the metallic nanoparticles in favour of the surface enhanced RAMAN scattering. Hence, the total fluorescence intensity is the result of only a part of the dye molecules situated in "active sites".

This can be nicely proved with a simple experiment. Using laser ablation, it is possible to selectively destroy the molecules that are situated between the nanoparticles (the presence of silver preventing the molecules that touch the nanoparticles to be destroyed). The fluorescence signal drops dramatically after this process. Only a SERS signal is still observable. 
It is possible to have a rough estimate of this active proportion from TEM images from which the average metal coverage associated to a nominal thickness deposition can be obtained. The proportion of "active sites" between the nanoparticles can be deduce to obtain the part of "active molecules" that do contribute to the fluorescence intensity, see figure 6 . For a silver thickness of $20 \mathrm{~nm}$, it appears that the global fluorescence enhancement factor of about 2 comes from only $20 \%$ of the molecules is local enhancement factor of about 10 !

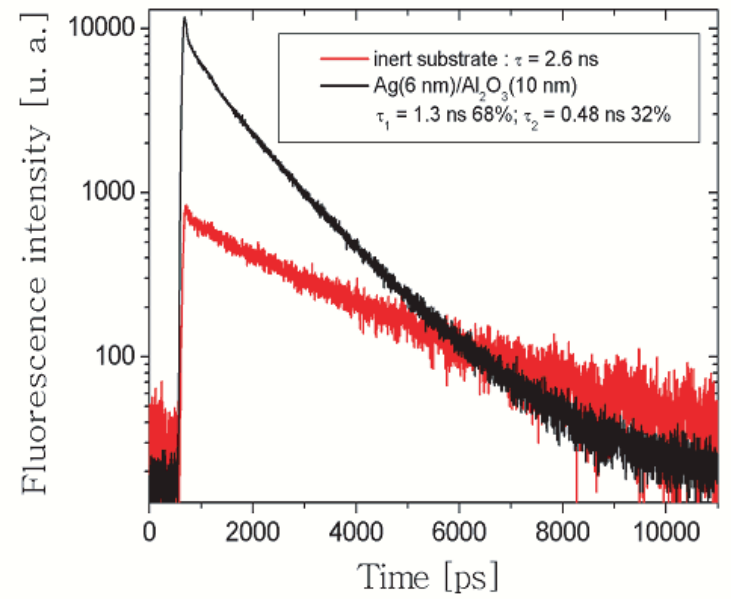

Figure 7. Fluorescence lifetime measurement of 0.01 monolayer of Rhodamine B adsorbed on a nanostructured slide and an inert slide.

A typical result is presented on figure 7 with the fluorescence decay of Rhodamine B deposited on a nanostructured silver sample with $6 \mathrm{~nm}$ thickness and a spacer layer of alumina of $10 \mathrm{~nm}$ and on an inert slide. The lifetime of the Rhodamine on an inert sample is $2.6 \mathrm{~ns}$, the fluorescence decay s mono-exponential. On the nanostructured sample, two decay components are visible corresponding to $\tau_{1}=1.3 \mathrm{~ns}(68 \%)$ and $\tau_{2}=0.48 \mathrm{~ns}(32 \%)$ leading to a mean lifetime value of nearly $1 \mathrm{~ns}$. The shortening of the fluorescence lifetime is a consequence of the presence of decay processes induced by the nanoparticle presence. The presence of multi-exponential is probably due to the various distances petween silver nanoparticles and molecules.

\section{CONCLUSION AND OUTLOOKS}

These results on the fluorescence enhancement are much promising for future biological applications in particular biochips since very large enhancement factors have been obtained with two photon excitation. The double gradient samples offer an easy way to determine the best enhancement parameters. Lifetime measurements are also promising not only as a mean to understand the coupling between the nanoparticle and the dye molecule but also as an alternative detection method for bio-chips for instance.

\section{REFERENCES}

1. J. Gersten and A. Nitzan, "Spectroscopic properties of molecules interacting with small dielectric particles," J. Chem. Phys. 75, pp. 1139-1152, August 1981.

2. J. P. Kottmann, O. J. F. Martin, D. R. Smith, and S. Schultz, "Field polarization and polarization charge distributions in plasmon resonant nanoparticles," New J. Phys. 2, p. 27, 2000. 
3. J. Kummerlen, A. Leitner, H. Brunner, F. Aussenegg, and A. Wokaun, "Enhanced dye fluorescence over silver island films : analysis of the distance dependence," Molecular Physics 80(5), pp. 1031$1046,1993$.

4. A. Wokaun, H. Lutz, A. King, U. Wild, and R. Ernst, "Energy transfer in surface enhanced luminescence," J. Chem Phys. 79(1), pp. 509-514, 1983.

5. D. Weitz, S. Garoff, J. Gertsen, and A. Nitzan, "The enhancement of raman scattering, resonance raman scattering and fluorescence from molecules adsorbed on a rough silver surface," J. Chem Phys. 78(9), pp. 5324-5338, 1983.

6. S. Lévêque-Fort et al., "Fluorescence enhancement near metallic nanoparticles under two photon excitation," Proc. SPIE 5139, pp. 20-26, October 2003.

7. G. Xu, M. Tazawa, P. Jin, S. Nakao, and K. Yoshimura, "Wavelength tuning of surface plasmon resonance using dielectric layers on silver island films," Appl. Phys. Lett. 82, pp. 3811-3813, June 2003.

8. S. Dutourtieux et al., "Near-field optical studies of semicontinuous metal films," Physical Review B 64, p. 1654053, 2001. 